\title{
BMJ Open Can physical assessment techniques aid diagnosis in people with chronic fatigue syndrome/myalgic encephalomyelitis? A diagnostic accuracy study
}

\author{
Lucy Hives, ${ }^{1}$ Alice Bradley, ${ }^{2}$ Jim Richards, ${ }^{3}$ Chris Sutton, ${ }^{4}$ James Selfe, ${ }^{5}$ \\ Bhaskar Basu, ${ }^{6}$ Kerry Maguire, ${ }^{7}$ Gail Sumner, ${ }^{8}$ Tarek Gaber, ${ }^{9}$ Annice Mukherjee, ${ }^{10}$ \\ Raymond N Perrin ${ }^{3}$
}

To cite: Hives L, Bradley A, Richards J, et al. Can physical assessment techniques aid diagnosis in people with chronic fatigue syndrome/myalgic encephalomyelitis? A diagnostic accuracy study. BMJ Open 2017;7:e017521. doi:10.1136/ bmjopen-2017-017521

- Prepublication history for this paper is available online. To view please visit the journal online (http://dx.doi.org/10. 1136/bmjopen-2017-017521).

Received 27 April 2017 Revised 23 August 2017 Accepted 29 September 2017

CrossMark

For numbered affiliations see end of article.

Correspondence to Professor Jim Richards; JRichards@uclan.ac.uk

\section{ABSTRACT}

Objective To assess five physical signs to see whether they can assist in the screening of patients with chronic fatigue syndrome/myalgic encephalomyelitis (CFS/ME) and potentially lead to quicker treatment.

Methods This was a diagnostic accuracy study with inter-rater agreement assessment. Participants recruited from two National Health Service hospitals, local CFS/ME support groups and the community were examined by three practitioners on the same day in a randomised order. Two allied health professionals (AHPs) performed independent examinations of physical signs including: postural/mechanical disturbances of the thoracic spine, breast varicosities, tender Perrin's point, tender coeliac plexus and dampened cranial flow. A physician conducted a standard clinical neurological and rheumatological assessment while looking for patterns of illness behaviour. Each examination lasted approximately $20 \mathrm{~min}$.

Results Ninety-four participants were assessed, 52 patients with CFS/ME and 42 non-CFS/ME controls, aged 18-60. Cohen's kappa revealed that agreement between the AHPs was substantial for presence of the tender coeliac plexus $(\kappa=0.65, p<0.001)$ and moderate for postural/mechanical disturbance of the thoracic spine $(\kappa=0.57, p<0.001)$ and Perrin's point $(\kappa=0.56$, $\mathrm{p}<0.001)$. A McNemar's test found no statistically significant bias in the diagnosis by the experienced AHP relative to actual diagnosis $(p=1.0)$ and a marginally non-significant bias by the newly trained AHP $(p=0.052)$. There was, however, a significant bias in the diagnosis made by the physician relative to actual diagnosis $(p<0.001)$, indicating poor diagnostic utility of the clinical neurological and rheumatological assessment.

Conclusions Using the physical signs appears to improve the accuracy of identifying people with CFS/ ME and shows agreement with current diagnostic techniques. However, the present study concludes that only two of these may be needed. Examining for physical signs is both quick and simple for the AHP and may be used as an efficient screening tool for CFS/ME. This is a small single-centre study, and therefore, further validation in other centres and larger populations is needed.
Strengths and limitations of this study

- This is the first study that explores agreement on the presence of physical signs in the screening of patients with chronic fatigue syndrome/myalgic encephalomyelitis (CFS/ME) and demonstrates proof of concept of these signs.

- This study did not assess the performance of physical signs in diagnosing CFS/ME among people reporting with illness in clinical practice.

- The screening method did not involve patient/family history, patient symptoms or any discussion between practitioner and patient; including these would be likely to increase accuracy in clinical practice.

- There were more female participants than males, at a rate similar to other studies, which have found a higher prevalence of CFS/ME in females; however, there were similar percentages of males and females among the controls.

- This was a small study that included only two allied health professionals using the Perrin technique and did not include participants with severe CFS/ME; this limits the generalisation of the findings.

\section{INTRODUCTION}

Chronic fatigue syndrome/myalgic encephalomyelitis (CFS/ME) is characterised by severe, debilitating fatigue that is exacerbated by exercise but does not improve with rest. This condition can lead to a substantial impairment, making every day activities difficult. There is currently no universally accepted method of diagnosing CFS/ME, so other conditions with a similar presentation of symptoms must first be ruled out. Therefore, the diagnosis of CFS/ME can often be a long process.

Up until recently, the most widely accepted criteria for CFS/ME was the revised US Centers for Disease Control and Prevention (CDC) definition, ${ }^{1}$ which required at least a 6-month period of fatigue that significantly 
interferes with a person's everyday activities. In addition to this, four or more of the following symptoms must have persisted or reoccurred within the last 6 months; impaired memory or concentration, postexertion malaise, sore throat, tender lymph nodes, aching or stiff muscles, joint pain, headache and unrefreshing sleep. The latest internationally recognised diagnostic criteria for CFS/ME is the International Consensus Criteria ${ }^{2}$ based on the widely adopted Canadian Criteria. ${ }^{3}$

The Canadian criteria included many of the cardiopulmonary and neurological abnormalities, which were not included in the CDC criteria. In addition, the Canadian criteria selected cases with less psychiatric comorbidity, more physical functional impairment, more fatigue/ weakness, plus neurological symptoms, which were significantly different from psychiatric controls with CFS/ME. ${ }^{4}$ However, in the UK, The National Institute for Health and Care Excellence (NICE) recognises the heterogeneity of the condition, advising that diagnosis of CFS/ME should be made after other possible diagnoses have been excluded and the symptoms have persisted for at least 4 months. The NICE guidance also states that diagnosis should be reassessed if none of the following key features of the disorder are present: postexertional fatigue, cognitive difficulties, sleep problems or chronic pain. ${ }^{5}$

Due to the heterogeneity of the disorder, the aetiology of CFS/ME remains unknown with many theories surrounding the pathophysiology of the disorder. ${ }^{6}$ The literature suggests that a range of possible causes including hormonal disturbances, immune system dysfunction, infectious and viral agents and nervous system abnormalities may all play a role in the pathophysiology of the disease. ${ }^{7}$ Early research suggested that infectious agents such as the Epstein-Barr virus (EBV) are associated with CFS/ME, with a persisting EBV infection being seen in those with the condition. ${ }^{8}$ A number of other infectious agents have been linked to the onset of CFS/ME including enteroviruses, which could explain the gastrointestinal symptoms often seen in patients, ${ }^{9}$ and also the acute $\mathrm{B} 19$ virus infection. ${ }^{10}$ It has been suggested that viral infections can alter immune response, which in turn can chronically activate the immune system ${ }^{11}$ and lead to many of the symptoms associated with CFS/ME. However, the research within this field is inconsistent with no evidence of a single infection causing CFS/ME, as well as many patients showing no sign of previous infection, ${ }^{12}$ suggesting that infectious agents may only be relevant to a subset of the patient population.

Lymphatic system alterations are suggested to have involvement in CFS/ME with dysfunction within the immune system, causing toxic build up within the central nervous system (CNS) and leading to engorgement of varicose lymph vessels that can be felt on examination. ${ }^{13}$ Tender lymph nodes are included in the ICC, Canadian and US CDC definitions ${ }^{1}$ and the NICE guidance, ${ }^{5}$ confirming it is a common symptom of the disorder related to immune system abnormalities. Techniques to target these engorgements and stimulate the drainage of toxins in the lymph nodes have been shown to lead to symptom improvement in patients with CFS/ME. ${ }^{13}$

Currently, there is no definitive way of diagnosing patients, although recent research has suggested that there is a link between CFS/ME, the lymphatic drainage system and the CNS and that, in fact, patients with CFS/ ME have certain physical signs present that may explain a number of the characteristics of the condition. ${ }^{13}$ The Perrin technique is a system of manual diagnosis and treatment that is based on the hypothesis that CFS/ME is a disorder of the lymphatic drainage of the CNS, which leads to five physical signs. ${ }^{14}$

The first aim of this study was to see whether the five physical signs of the Perrin technique can assist in the screening of patients with CFS/ME, which could then subsequently lead to quicker treatment. Second, the study aimed to see whether the diagnostic accuracy was similar for a newly trained allied health professional (AHP) with no prior experience of CFS/ME compared with an experienced AHP.

\section{METHODS}

This was a diagnostic accuracy study with inter-rater agreement assessment. R\&D approval was obtained from each participating NHS Trust. The full study protocol has been made available at the same publisher.

\section{Recruitment}

Participants with CFS/ME were recruited from two hospital clinics and local support groups within the North West. Social media and posters displayed around the University of Central Lancashire were used to advertise the study. Healthy participants were recruited from non-blood relatives and friends of people with CFS/ME, staff and students from the university and from those who had heard about the study over social media. All participants voluntarily contacted the research team, by email, telephone or post, to register their interest.

Those who contacted the researcher were sent a participant information sheet via email or post. Potential participants were given time to consider participation, during which they could contact the researcher to ask any questions about the research. The researcher then sent out a consent form to each person, which were then returned if they were happy to take part.

\section{Participant eligibility}

On receiving their completed consent form, the researcher contacted each person by telephone in order to assess their eligibility to take part in the study. Potential participants who were aged between 18 and 60 were assessed using two forms: a recruitment screening form based on the NICE guidance ${ }^{5}$ and a form based on the International Consensus Criteria ${ }^{2}$ (reference standard). These eligibility criteria were used to ensure that each patient with CFS/ME had received a correct diagnosis of 
CFS/ME and to ensure that control participants did not have undiagnosed CFS/ME.

\section{Inclusion criteria for CFS/ME group}

To be included, patients with CFS/ME needed to have a prior formal diagnosis of CFS $/ \mathrm{ME}^{5}$ at a National Health Service (NHS) hospital specialised clinic, persistent or recurrent fatigue for at least the past 6 months, a clear starting point to the fatigue, the fatigue should be unexplained by any other conditions, the fatigue should reduce the amount of physical activity each person could do and the fatigue should feel worse after physical activity.

Additionally, patients with CFS/ME needed to have at least one of the following symptoms: difficulty sleeping or insomnia, joint pain without swelling, headaches, painful lymph nodes that are not enlarged, recurrent sore throats, muscle pain without swelling, poor mental function (eg, difficulty thinking), symptoms getting worse after physical or mental exertion, feeling unwell or having flu-like symptoms, dizziness or nausea or heart palpitations.

\section{Exclusion criteria for both CFS/ME and control groups}

The following were excluded from taking part: people needing to use a wheelchair and pregnant and lactating women. In addition: comorbidities including: anaemias, autoimmune diseases, cardiac disease, endocrine disorders, infectious diseases, intestinal diseases, malignancies, neurological disorders, primary psychiatric disorders, significant pulmonary disease and primary sleep disorders. Additional exclusion criteria for control group included: a diagnosis of CFS/ME or a family history of $\mathrm{CFS} / \mathrm{ME}$.

Each participant was allocated a participant ID number with the clinical team being blinded to the groupings.

\section{Assessment methods}

Perrin technique

The examination comprised the following four assessments:

A. Participant standing: observation and palpation of thoracic spine for any postural defects; regions of redness, temperature change or skin rashes or eruptions, for example, acne/boils.

B. Participant lying supine: observation and palpation of breast tissue for varicosities in the surface lymphatics and abnormal breast tenderness at 'Perrin's point', which is a superficial tender area found at around $2-3 \mathrm{~cm}$ lateral and superior to the left nipple. ${ }^{15}$

C. With the participant remaining supine, palpation of the region of the coeliac plexus just below the xiphoid in the upper central area of the abdomen for any abnormal tenderness with possible temperature change in the region.

D. With the participant remaining in a supine position, cradle the head and examine the quality of the cranial rhythmic impulse. ${ }^{16}$

These assessments resulted in identification of the following signs as present or not present: (1) postural/ mechanical disturbances of the thoracic spine (assessment A); (2) breast varicosities (assessment B); (3) tender Perrin's point (assessment B); (4) tender coeliac plexus (assessment $\mathrm{C}$ ); and (5) dampened cranial flow (assessment D).

If all five signs were present, then the participant is classified as having CFS/ME. If one or more of the signs was absent, then the participant is classified as not having $\mathrm{CFS} / \mathrm{ME} .^{16}$

The examination was performed by two AHPs. One had 10 years of experience of using the Perrin technique and working with patients with CFS/ME (experienced AHP); the other was newly trained in the Perrin technique with no prior experience of CFS/ME (newly trained AHP). The newly trained AHP received training, especially for this study, which involved being taught how to examine patients for the five physical signs and having hand-on experience of practising the technique.

\section{Rheumatological assessment}

A standard clinical neurological and rheumatological assessment was performed by a physician while observing the participant for any signs of illness behaviour, but no clinical history was taken. The neurological examination included muscle strength testing, examination of muscle tone in arms and legs, coordination including the finger nose test, heel-shin test, heel-toe walking, reflexes and sensation with eyes closed. The rheumatological examination examined joint swelling, wasting of regional muscles, deformity of joint, redness in joints or tendons and the palpation of the margin of joints in hands and feet.

If all the tests were normal with no observed illness behaviour, the patient was classified as not having CFS/ ME, whereas if abnormal observations were made, the physician used their clinical experience to decide if the participant had CFS/ME. The physician performing these assessments had experience of working in NHS clinics for CFS/ME but had no experience of the Perrin technique (physician).

\section{Data collection}

For each participant, all data collection was performed on a single assessment day. They were allowed to bring a friend or relative along for support or, if requested, a chaperone was provided by the research team. Participants were rebriefed on what would be involved, and consent was confirmed.

Each of the participants were separately examined by the three practitioners in different rooms. The order of examinations was randomised. No conversation took place between the participants and practitioners except to determine if there was any pain or tenderness in certain regions.

\section{Data analysis}

A priori, the Perrin technique required all five symptoms to be present for a patient to be diagnosed as CFS/ME. Using this criterion, the sensitivity and specificity of the 


\begin{tabular}{lcc|}
\hline Table 1 & Gender balance of the two groups \\
\hline & CFS/ME & Control \\
\hline Male & $9(17 \%)$ & $25(60 \%)$ \\
Female & $43(83 \%)$ & $17(40 \%)$
\end{tabular}

CFS/ME, chronic fatigue syndrome/myalgic encephalomyelitis.

diagnosis of CFS/ME relative to the reference standard were estimated as simple proportions, accompanied by exact (binomial) 95\% CIs. McNemar's test was used to investigate whether any of the practitioners systematically underdiagnosed or overdiagnosed CFS/ME. Agreement in the diagnosis of CFS/ME between AHPs using the Perrin technique was estimated using Cohen's kappa ( $\kappa)$ coefficient; an approximate $95 \%$ CI for $\kappa$ was obtained using bias-corrected non-parametric bootstrapping. Agreement between their identification of the individual physical signs was also estimated using the same methods.

Exploratory analysis of the performance of using different numbers of symptoms was then performed. Symptoms were removed based on the observed agreement between AHPs; thus, the four-symptom test excluded the symptom with lowest agreement, the three-symptom test excluded the two symptoms with the two lowest agreements and so on.

\section{Sample size}

The target sample size was 50 with CFS/ME and 50 controls to enable estimation of sensitivity and specificity of each Perrin technique AHP's diagnosis of CFS/ ME (relative to the imperfect reference standard) to within $\pm 9.9 \%$ with $95 \%$ confidence if the sensitivity and specificity were each at least $85 \%$. It would also enable the estimation of $\kappa$ (for inter-rater agreement between each pair), with $95 \%$ confidence, to within \pm 0.140 providing $\kappa$ were at least 0.7 .

\section{RESULTS}

Ninety-four participants were recruited: 52 patients with $\mathrm{CFS} / \mathrm{ME}$ and 42 non-CFS/ME controls. The gender ratio in the CFS/ME group (shown in table 1) is in keeping with epidemiological studies, which have shown a larger number of patients with CFS/ME to be female with a ratio of 2:1 or more. ${ }^{17}$ Results show that, on average, the experienced AHP was most accurate $(86 \%)$ at correctly diagnosing participants. This was followed by the newly trained who correctly diagnosed $77 \%$ and the physician who correctly diagnosed $69 \%$ of participants.

\section{Sensitivity and specificity}

Table 2 shows the prevalence, sensitivity (the proportion of positive results in people with CFS/ME), specificity (the proportion of negative CFS/ME results in healthy controls), positive predictor value (the proportion with CFS/ME in participants with a positive result) and negative predictive value (the proportion of healthy controls with negative CFS/ME results).

When using the five physical signs of the Perrin technique, the sensitivity for the experienced AHP was 0.88 (95\% CI 0.77 to 0.96$)$ and the specificity was 0.83 (95\% CI 0.69 to 0.93$)$. Similarly, when the newly trained AHP used the same technique, the specificity was $0.86(95 \%$ CI 0.71 to 0.95 ); however, sensitivity was lowered to 0.69 (95\% CI 0.55 to 0.81 ). This shows that although using the same technique, the newly trained AHP struggled more frequently to identify correctly all five physical signs in people with a positive diagnosis of CFS/ME. There was no statistically significant bias in the diagnosis by the experienced AHP relative to actual diagnosis $(p=1.0)$. There was also a marginally non-significant evidence of biased diagnosis by the newly trained AHP relative to actual diagnosis $(\mathrm{p}=0.052)$, potentially favouring a non-CFS/ME diagnosis.

When using the standard clinical neurological and rheumatological examination, the sensitivity of the physician was 0.44 (95\% CI 0.30 to 0.59 ) and the specificity was 1.0 (95\% CI 0.92 to 1.0$)$. These results show that while able to identify correctly all healthy controls, the physician struggled the most out of all three practitioners to identify correctly people with a positive diagnosis of $\mathrm{CFS} / \mathrm{ME}$. There was a significant bias in the diagnosis by the physician relative to actual diagnosis $(\mathrm{p}<0.001)$, also favouring a non-CFS diagnosis.

\section{Agreement between the experienced and newly trained AHPs}

There was moderate agreement between the experienced and newly trained AHPs on overall diagnosis using the five physical signs of the Perrin technique $(\kappa=0.56,95 \%$ CI 0.40 to $0.72, \mathrm{p}<0.001)$. Regarding the identification of the individual physical signs, there was substantial agreement between the AHPs on the presence of the tender coeliac plexus $(\kappa=0.65 ; 95 \%$ CI 0.48 to $0.80, \mathrm{p}<0.001)$ and agreement was moderate both on

Table 2 Diagnostic performance of the three practitioners

\begin{tabular}{|c|c|c|c|c|c|c|c|c|c|}
\hline Practitioner & $\begin{array}{l}\text { True } \\
\text { positives }\end{array}$ & $\begin{array}{l}\text { False } \\
\text { negatives }\end{array}$ & $\begin{array}{l}\text { False } \\
\text { positives }\end{array}$ & $\begin{array}{l}\text { True } \\
\text { negatives }\end{array}$ & Sensitivity & Specificity & $\begin{array}{l}\text { Positive } \\
\text { predictive } \\
\text { value }\end{array}$ & $\begin{array}{l}\text { Negative } \\
\text { predictive } \\
\text { value }\end{array}$ & $\begin{array}{l}\text { Accuracy } \\
(\%)\end{array}$ \\
\hline Experienced AHP & 46 & 6 & 7 & 35 & 0.88 & 0.83 & 0.87 & 0.85 & 86.2 \\
\hline Newly trained AHP & 36 & 16 & 6 & 36 & 0.69 & 0.86 & 0.86 & 0.69 & 76.6 \\
\hline Physician & 23 & 29 & 0 & 42 & 0.44 & 1.00 & 1.00 & 0.57 & 69.1 \\
\hline
\end{tabular}

AHP, allied health professional. 
the presence of postural/mechanical disturbance of the thoracic spine $(\kappa=0.57 ; 95 \%$ CI 0.39 to $0.73, \mathrm{p}<0.001)$ and on the presence of Perrin's point $(\kappa=0.56$; $95 \%$ CI 0.37 to $0.73, \mathrm{p}<0.001)$. However, there was only fair agreement between the AHP's identification of the dampened cranial flow ( $\kappa=0.35 ; 95 \%$ CI 0.15 to $0.54, \mathrm{p}=0.001$ ), and there was non-significant 'slight' agreement on the presence of breast varicosities $(\kappa=0.03 ; 95 \%$ CI -0.12 to 0.22 , $\mathrm{p}=0.75)$.

\section{Diagnostic properties of alternative criteria}

Given the relatively low sensitivity for the newly trained AHP, alternative criteria were applied using lower number of physical signs. Using the kappa statistics for agreement between the experienced and newly trained AHPs on the presence of the Perrin technique physical signs, each physical sign, in order starting with the sign with the least agreement, was excluded and sensitivity and specificity were recalculated. Overall, the AHPs were more accurate at diagnosing participants when using only two of the five signs (tender coeliac plexus and postural/mechanicaldisturbance of the thoracic spine). Tables 3 and 4 show the accuracy of the experienced and newly trained AHPs, respectively, when using each number of physical signs. The accuracy of the experienced AHP is the same using 3-5 of the physical signs $(86.2 \%)$ with the highest accuracy using only 2 of the physical signs $(88.3 \%)$. The accuracy of the newly trained AHP is highest using only one or two of the physical signs $(80.9 \%)$. Therefore, accuracy for both AHPs, overall, is highest when using only tests of tender coeliac plexus and postural/mechanical disturbance of the thoracic spine.

Cohen's $\kappa$ was recomputed for the level of agreement between the experienced and newly trained AHPs on whether they believed that the 94 individuals had CFS/ ME or were healthy controls, using the reduced two physical signs of the Perrin technique. There was substantial agreement between the two AHPs on overall diagnosis using the two physical signs, $\kappa=0.61$ (95\% CI 0.45 to 0.74 ), $\mathrm{p}<0.001$. There was no statistically significant bias in the diagnosis by the experienced AHP and actual diagnosis $(\mathrm{p}=0.55)$ or by the newly trained AHP and actual diagnosis $(\mathrm{p}=0.63)$ when using the reduced two-item Perrin technique, showing that the revised criteria no longer favoured a non-CFS/ME diagnosis.

\section{DISCUSSION}

Between the AHPs, the AHP with prior experience of using the Perrin technique was the most accurate at correctly diagnosing individuals with $\mathrm{CFS} / \mathrm{ME}$, whereas the AHP with no prior experience of CFS/ME or the Perrin technique was better at correctly recognising healthy individuals.

The AHP experienced in the Perrin technique was able to identify $88 \%$ of patients with CFS/ME using all

Table 3 Experienced allied health professional

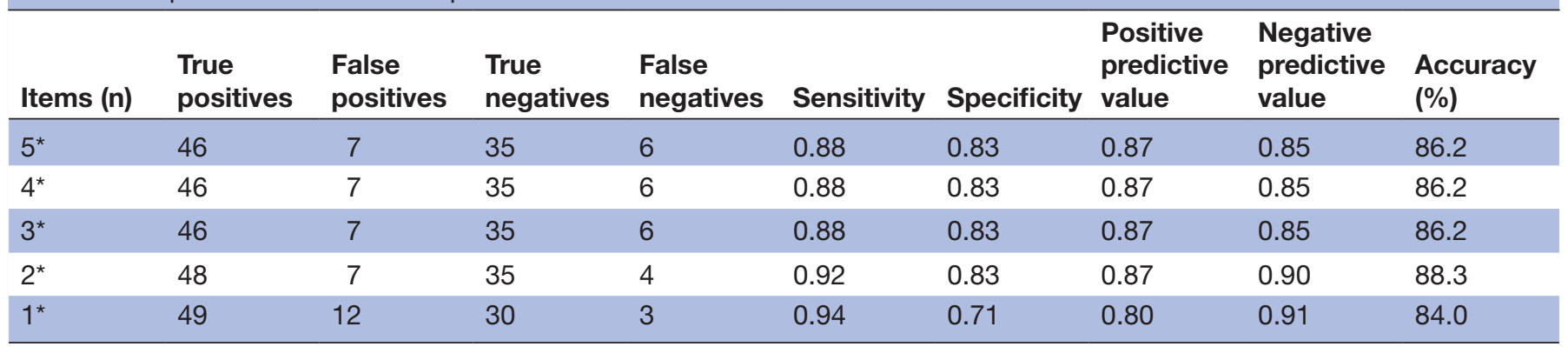

5* includes all 5 Perrin technique physical signs; $4^{*}$ includes postural/mechanical disturbances of the thoracic spine, tender Perrin's point, tender coeliac plexus and dampened cranial flow; $3^{*}$ includes postural/mechanical disturbances of the thoracic spine, tender Perrin's point and tender coeliac plexus; $2^{*}$ includes tender coeliac plexus and postural/mechanical disturbance of the thoracic spine; and $1^{*}$ includes tender coeliac plexus.

\begin{tabular}{|c|c|c|c|c|c|c|c|c|c|}
\hline Items (n) & $\begin{array}{l}\text { True } \\
\text { positives }\end{array}$ & $\begin{array}{l}\text { False } \\
\text { positives }\end{array}$ & $\begin{array}{l}\text { True } \\
\text { negatives }\end{array}$ & $\begin{array}{l}\text { False } \\
\text { negatives }\end{array}$ & Sensitivity & Specificity & $\begin{array}{l}\text { Positive } \\
\text { predictive value }\end{array}$ & $\begin{array}{l}\text { Negative } \\
\text { predictive value }\end{array}$ & $\begin{array}{l}\text { Accuracy } \\
(\%)\end{array}$ \\
\hline $5^{*}$ & 36 & 6 & 36 & 16 & 0.69 & 0.86 & 0.86 & 0.69 & 76.6 \\
\hline $4^{*}$ & 36 & 7 & 35 & 16 & 0.69 & 0.83 & 0.84 & 0.69 & 75.5 \\
\hline $3^{*}$ & 40 & 7 & 35 & 12 & 0.77 & 0.83 & 0.85 & 0.74 & 79.8 \\
\hline $2^{*}$ & 42 & 8 & 34 & 10 & 0.81 & 0.81 & 0.84 & 0.77 & 80.9 \\
\hline $1^{*}$ & 48 & 14 & 28 & 4 & 0.92 & 0.67 & 0.77 & 0.88 & 80.9 \\
\hline
\end{tabular}

$5^{\star}$ includes all 5 Perrin technique physical signs; $4^{\star}$ includes postural/mechanical disturbances of the thoracic spine, tender Perrin's point, tender coeliac plexus and dampened cranial flow; $3^{*}$ includes postural/mechanical disturbances of the thoracic spine, tender Perrin's point and tender coeliac plexus; $2^{*}$ includes tender coeliac plexus and postural/mechanical disturbance of the thoracic spine; and $1^{*}$ includes tender coeliac plexus. 
five physical signs and $83 \%$ of healthy controls who did not display all five signs. However, for the AHP with no prior Perrin technique experience, they were able to detect $86 \%$ of healthy controls but identified only $69 \%$ of patients with CFS/ME using all five signs; there was borderline non-significant evidence of this AHP underdiagnosing rather than overdiagnosing CFS/ME. This highlights that the newly trained AHP was not able to identify all five signs in some people with a diagnosis of CFS/ME. However, this does not necessarily mean that the signs were not present; it could mean that the newly trained AHP found these signs more difficult to detect. Despite this, there was moderate agreement between both AHPs on overall diagnosis.

The physician was able to correctly identify $100 \%$ of the healthy controls using the standard clinical neurological and rheumatological examination. However, they were only able to correctly identify $44 \%$ of patients with $\mathrm{CFS} / \mathrm{ME}$, and the tendency to underdiagnose CFS/ME was highly statistically significant $(p<0.001)$. This affirms the current approach used in CFS diagnostics based on NICE guidance in that clinical examination is most useful in identifying alternative diagnoses and to exclude the diagnosis of CFS/ME, but that clinical examination is not a useful modality for confirming diagnosis of CFS/ME.

The agreement of the AHPs on the presence of each of the five physical signs varied from substantial agreement on the presence of the tender coeliac plexus to non-significant 'slight' agreement on the presence of breast varicosities. From the results presented, it would seem that the physical signs can improve the accuracy of diagnosing CFS/ME, although not all of the five physical signs may be necessary. Even with the experienced AHP, who, on the whole, was able to identify the five signs, breast varicosities and dampened cranial flow did not improve accuracy of diagnosis. Further exploration of the sensitivity, specificity and accuracy found that using only two of the five physical signs (tender coeliac plexus and postural/mechanical disturbance of the thoracic spine) was the most accurate and efficient method of correctly diagnosing the participants for both AHPs despite their differing levels of prior experience of CFS/ME and the Perrin technique.

Previous work ${ }^{15}$ found Perrin's point to have a diagnostic accuracy of $80 \%$ in patients with CFS/ME. This was very similar to the accuracy when including Perrin's point in the current study (accuracy: experienced $\mathrm{AHP}=86.2 \%$ and newly trained $\mathrm{AHP}=79.8 \%$ ). However, it was found that the omission of Perrin's point marginally increased the accuracy of the AHP with prior experience of the Perrin technique by $2.1 \%$ and the AHP with no prior experience of the Perrin technique by $1.1 \%$.

\section{Limitations and suggestions for future research}

Although this study shows clearly that diagnostic accuracy for CFS/ME increases using the physical signs of the Perrin technique, there are some limitations, which should be highlighted. First, this study recruited two groups of participants, people with a prior diagnosis of CFS/ME and healthy controls with no symptoms of the condition, as the purpose was to establish 'proof of concept' of the Perrin technique. Therefore, the study did not explore how accurate the Perrin technique physical signs would be, when presented with patients with different conditions with similar presenting symptoms such as fibromyalgia. However, in a clinical setting, knowing the history and symptoms together with the physical signs would help to differentially diagnose CFS/ME from other possible illnesses. Now that we have established proof-of-concept, it will be important to identify whether the physical signs of the Perrin technique, combined with history, improve the accuracy of identifying people with chronic fatigue syndrome/myalgic encephalomyelitis (CFS/ME) in clinical practice.

The gender balance in the patients with CFS/ME was similar to that of previously published work, ${ }^{17}$ although the healthy volunteers were recruited as a convenience sample, which was not gender matched. Therefore, any prior knowledge of the expected male to female ratio among patients with CFS/ME could have influenced the results. It should be noted, however, that none of the practitioners knew if the control group was matched or not for gender and that the AHP with no prior experience of CFS/ME was unaware of the gender balance. Although all participants were aged between 18 and 60, individual age data were not collected for each participant. However, it is reported that CFS/ME develops more commonly in those between their mid-twenties and mid-forties. ${ }^{18}$

Future research should investigate whether the physical signs are more apparent in people with more severe CFS/ME. The present study did not collect data on how long each participant had had CFS/ME for or on the severity of their symptoms, which could add further understanding.

Only three practitioners (one in each category) were used. We therefore have very limited information on agreement between practitioners and whether diagnostic accuracy is substantially affected by experience; we have no information on within-category variation. Future research should involve a study design whereby there are multiple experienced AHPs, newly trained AHPs and physicians.

A further limitation is that we selected specific symptoms for exclusion from the diagnostic criterion based on the agreement between practitioners. This was a pragmatic decision based on the estimated agreement between practitioners; it does not invalidate our findings, but there may be alternative criteria that have better performance. Again, optimisation of the set of symptoms for diagnosis merits further investigation in a larger study, in which additional information around acceptability and performance of individual physical sign assessments could be performed. 


\section{CONCLUSION}

Current methods for diagnosing CFS/ME are challenging. The use of standard clinical neurological and rheumatological examination to examine illness behaviour is more likely to have a false negative result than a true positive one. Using certain physical signs appears to improve the accuracy of identifying people with CFS/ME and shows agreement with current diagnostic techniques, although not all of the physical signs were useful, and it is suggested that only two of these are needed. Examining for physical signs is both quick and simple for the AHP and may be used as an efficient screening tool for CFS/ ME. This study did not include patient/family history or the patient talking about their symptoms, which should increase accuracy in clinical practice.

\section{Author affiliations}

${ }^{1}$ School of Dentistry, University of Central Lancashire, Preston, UK

${ }^{2}$ Department of Neurophysiology, Lancashire Teaching Hospitals NHS Foundation

Trust, Preston, UK

${ }^{3}$ Allied Health Research Unit, University of Central, Lancashire, UK

${ }^{4}$ Lancashire Clinical Trials Unit, University of Central Lancashire, Preston, UK

${ }^{5}$ Department of Health Professions, Manchester Metropolitan University,

Manchester, Greater Manchester, UK

${ }^{6}$ University Hospital of South Manchester NHS Foundation Trust/ Central,

Manchester University Hospitals NHS Foundation Trust, Rehabilitation Medicine,

Manchester, UK

${ }^{7}$ Department of Osteopathy, The Good Health Centre, County Clare, Ireland

${ }^{8}$ Back to Health Physiotherapy, Bolton, UK

${ }^{9}$ Taylor Rehabilitation Unit leigh, Leigh Infirmary, Leigh, Greater Manchester, UK

${ }^{10}$ Department of Endocrinology, Salford Royal NHS Foundation Trust, Salford, UK

Contributors LJH recruited participants, collected data and analysed the data. ARB recruited participants and collected data. JR initiated the project, designed the research methods and analysed the data. CS wrote the statistical analysis plan and analysed the data. JS initiated the project and designed the research methods. BB, KM and GS collected data. TG and AM recruited participants. RNP initiated the project and designed the research methods. All authors drafted and revised the paper.

Funding This work was supported by The Fund for 0steopathic Research into Myalgic Encephalomyelitis (registered charity number: 1045005).

Competing interests This research explored the findings by one of the co-authors, RNP. To avoid any conflict of interest, he was not involved in any of the recruitment of participants, clinical examinations, data collection or analysis. RNP's role in the study was to assist with developing the design, writing the protocol, setting up the project coordinating committee with the different clinical recruitment centres, applying for ethical approval and assisting with the writing of the introduction and methods of the paper. There were no other conflicts of interest.

Ethics approval NRES Committee North West-Lancaster (REC reference 12/ NW/0877).

Provenance and peer review Not commissioned; externally peer reviewed.

Data sharing statement The relevant anonymised patient level data are available on request from the corresponding author.
Open Access This is an Open Access article distributed in accordance with the Creative Commons Attribution Non Commercial (CC BY-NC 4.0) license, which permits others to distribute, remix, adapt, build upon this work non-commercially, and license their derivative works on different terms, provided the original work is properly cited and the use is non-commercial. See: http://creativecommons.org/ licenses/by-nc/4.0/

(c) Article author(s) (or their employer(s) unless otherwise stated in the text of the article) 2017. All rights reserved. No commercial use is permitted unless otherwise expressly granted.

\section{REFERENCES}

1. Fukuda K. The chronic fatigue syndrome: a comprehensive approach to its definition and study. Ann Intern Med 1994;121:953-9.

2. Carruthers BM, van de Sande MI, De Meirleir KL, et al. Myalgic encephalomyelitis: international consensus criteria. J Intern Med 2011;270:327-38.

3. Carruthers BM. Definitions and aetiology of myalgic encephalomyelitis: how the Canadian consensus clinical definition of myalgic encephalomyelitis works. J Clin Pathol 2007;60:117-9.

4. Jason LA, Richman JA, Rademaker AW, et al. A communitybased study of chronic fatigue syndrome. Arch Intern Med 1999;159:2129-37.

5. National Institute for Health and Care Excellence. Chronic fatigue syndrome/myalgic encephalomyelitis (or encephalopathy): diagnosis and management of chronic fatigue syndrome/myalgic encephalomyelitis (or encephalopathy) in adults and children. London: NICE, 2007.

6. Bansal AS, Bradley AS, Bishop KN, et al. Chronic fatigue syndrome, the immune system and viral infection. Brain Behav Immun 2012;26:24-31.

7. Shephard RJ. Chronic fatigue syndrome. Sports Med 2001;31:167-94.

8. Straus SE, Tosato G, Armstrong G, et al. Persisting illness and fatigue in adults with evidence of epstein-barr virus infection. Ann Intern Med 1985;102:7-16.

9. Chia JK, Chia AY. Chronic fatigue syndrome is associated with chronic enterovirus infection of the stomach. J Clin Pathol 2008;61:43-8.

10. Kerr JR, Bracewell J, Laing I, et al. Chronic fatigue syndrome and arthralgia following parvovirus B19 infection. J Rheumatol 2002;29:595-602.

11. Lorusso L, Mikhaylova SV, Capelli E, et al. Immunological aspects of chronic fatigue syndrome. Autoimmun Rev 2009;8:287-91.

12. Afari N, Buchwald D. Chronic fatigue syndrome: a review. Am J Psychiatry 2003;160:221-36.

13. Perrin RN, Richards JD, Pentreath V, et al. Muscle fatigue in chronic fatigue syndrome/myalgic encephalomyelitis (CFS/ME) and its response to a manual therapeutic approach: a pilot study. Int $J$ Osteopath Med 2011;14:96-105.

14. Perrin R. The involvement of cerebrospinal fluid and lymphatic drainage in chronic fatigue syndrome/ME. PhD [thesis]. University of Salford: Salford, 2005.

15. Puri BK, Gunatilake KD, Fernando KA, et al. Increased tenderness in the left third intercostal space in adult patients with myalgic encephalomyelitis: a controlled study. J Int Med Res 2011;39:212-4.

16. Perrin RN. Lymphatic drainage of the neuraxis in chronic fatigue syndrome: a hypothetical model for the cranial rhythmic impulse. $J$ Am Osteopath Assoc 2007;107:218-24.

17. Jason LA, Richman JA, Rademaker AW, et al. A communitybased study of chronic fatigue syndrome. Arch Intern Med 1999;159:2129-37.

18. NHS Choices. Chronic fatigue syndrome. http://www.nhs.uk/ conditions/Chronic-fatigue-syndrome/Pages/Introduction.aspx. (accessed 18 Aug 2017). 